PROCEEDINGS OF THE

AMERICAN MATHEMATICAL SOCIETY

Volume 137, Number 8, August 2009, Pages 2503-2515

S 0002-9939(09)09881-5

Article electronically published on March 24, 2009

\title{
A VARIATION OF MULTIPLE $L$-VALUES ARISING FROM THE SPECTRAL ZETA FUNCTION OF THE NON-COMMUTATIVE HARMONIC OSCILLATOR
}

\author{
KAZUFUMI KIMOTO AND YOSHINORI YAMASAKI
}

(Communicated by Wen-Ching Winnie Li)

\begin{abstract}
A variation of multiple $L$-values, which arises from the description of the special values of the spectral zeta function of the non-commutative harmonic oscillator, is introduced. In some special cases, we show that its generating function can be written in terms of the gamma functions. This result enables us to obtain explicit evaluations of them.
\end{abstract}

\section{INTRODUCTION}

The multiple zeta values

$$
\zeta_{k}^{\bullet}\left(n_{1}, \ldots, n_{k}\right):=\sum_{1 \leq i_{1}<\cdots<i_{k}} \frac{1}{i_{1}^{n_{1}} i_{2}^{n_{2}} \ldots i_{k}^{n_{k}}}
$$

are natural extensions of the Riemann zeta value $\zeta(n)=\sum_{i=1}^{\infty} i^{-n}$ introduced by Euler and have been of continuing interest to many mathematicians [18. Recently, it has been shown by several authors that they appear in various fields in mathematics such as knot invariant theory, quantum group theory and mathematical physics (see, e.g., [11, 20]). This fact implies the richness of the theory of multiple zeta values and has encouraged recent studies of them. One of the main problems in studying multiple zeta values is to clarify the $\mathbb{Q}$-algebra structure of the space spanned by them, which is closely related to that of the category of mixed Tate motives. In fact, for this purpose, many results concerning relations among them and exact calculations of them are investigated. Furthermore, as a natural

Received by the editors May 8, 2008.

2000 Mathematics Subject Classification. Primary 11M41, 05E05; Secondary 11P81, 05A15.

Key words and phrases. Multiple zeta values, multiple $L$-values, Bernoulli numbers, noncommutative harmonic oscillator, spectral zeta function, symmetric functions.

The first author was supported in part by Grant-in-Aid for Young Scientists (B) No. 20740021.

The second author was supported in part by Grant-in-Aid for JSPS Fellows No. 19002485.

(C)2009 American Mathematical Society Reverts to public domain 28 years from publication 
generalization, Arakawa and Kaneko [2] introduce two kinds of multiple $L$-values:

$$
\begin{aligned}
L_{\text {III }}\left(n_{1}, \ldots,\right. & \left.n_{k} ; f_{1}, \ldots, f_{k}\right) \\
& :=\sum_{m_{1}>\cdots>m_{k}>0} \frac{f_{1}\left(m_{1}-m_{2}\right) \ldots f_{k-1}\left(m_{k-1}-m_{k}\right) f_{k}\left(m_{k}\right)}{m_{1}^{n_{1}} m_{2}^{n_{2}} \ldots m_{k}^{n_{k}}}, \\
L_{*}\left(n_{1}, \ldots,\right. & \left.n_{k} ; f_{1}, \ldots, f_{k}\right) \\
& :=\sum_{m_{1}>\cdots>m_{k}>0} \frac{f_{1}\left(m_{1}\right) f_{2}\left(m_{2}\right) \ldots f_{k}\left(m_{k}\right)}{m_{1}^{n_{1}} m_{2}^{n_{2}} \ldots m_{k}^{n_{k}}},
\end{aligned}
$$

where $f_{1}, \ldots, f_{k}$ are $\mathbb{C}$-valued periodic functions on $\mathbb{Z}$, and they also study their relations and exact evaluations.

In this paper, we study the following variation $S_{k}^{(N, M)}\left(n_{1}, \ldots, n_{k}\right)(N, M \in \mathbb{N})$ of the multiple $L$-values:

$$
S_{k}^{(N, M)}\left(n_{1}, \ldots, n_{k}\right):=\sum_{1 \leq i_{1} \leq i_{2} \leq \cdots \leq i_{k}} \varepsilon_{i_{1} i_{2} \ldots i_{k}}^{(N)} \frac{\omega_{M}^{i_{1}+i_{2}+\cdots+i_{k}}}{i_{1}^{n_{1}} i_{2}^{n_{2}} \ldots i_{k}^{n_{k}}},
$$

where $\omega_{M}$ is a primitive $M$ th root of unity and

$$
\begin{aligned}
\varepsilon_{i j}^{(N)} & :=\left\{\begin{array}{ll}
0 & i=j \not \equiv 0 \\
1 & \text { otherwise }
\end{array} \quad(\bmod N)=1-\delta_{i j}\left(1-\frac{1}{N} \sum_{r=0}^{N-1} \omega_{N}^{r i}\right),\right. \\
\varepsilon_{i_{1} i_{2} \ldots i_{k}}^{(N)} & :=\prod_{j=1}^{k-1} \varepsilon_{i_{j} i_{j+1}}^{(N)} .
\end{aligned}
$$

Here $\delta_{i j}$ is the Kronecker delta. For simplicity, we sometimes write

$$
\begin{aligned}
S_{k}^{(N)}\left(n_{1}, \ldots, n_{k}\right) & =S_{k}^{(N, N)}\left(n_{1}, \ldots, n_{k}\right), \\
S_{k}^{(N, M)}(n) & =S_{k}^{(N, M)}(n, \ldots, n), \\
S_{k}^{(N)}(n) & =S_{k}^{(N)}(n, \ldots, n) .
\end{aligned}
$$

We note that $S_{1}^{(N, M)}(n)=L i_{n}\left(\omega_{M}\right)$, where $L i_{n}(z):=\sum_{i=1}^{\infty} z^{i} / i^{n}$ is the polylogarithm.

The aim of the paper is to establish generating function formulas for the series $S_{k}^{(N, M)}(n)$, and give an explicit evaluation of them in terms of Bernoulli numbers in the special case where $N=M=2$ and $n$ is even. It is quite remarkable that the values $S_{k}^{(2)}(n)$ can be fully computable; in fact, there are few examples of computable multiple $L$-values. In this sense, $S_{k}^{(N)}(n)$ seems to be a nice variant of the ordinary multiple $L$-values.

We will sometimes refer to $S_{k}^{(N, M)}\left(n_{1}, \ldots, n_{k}\right)$ as a partial multiple $L$-value because it is indeed a partial sum of the "non-strict" multiple $L$-value

$$
\sum_{1 \leq i_{1} \leq i_{2} \leq \cdots \leq i_{k}} \frac{\omega_{M}^{i_{1}+i_{2}+\cdots+i_{k}}}{i_{1}^{n_{1}} i_{2}^{n_{2}} \ldots i_{k}^{n_{k}}}=S_{k}^{(1, M)}\left(n_{1}, n_{2}, \ldots, n_{k}\right) .
$$

In particular, $S_{k}^{(1)}\left(n_{1}, n_{2}, \ldots, n_{k}\right)$ gives the non-strict multiple zeta value (see, e.g., [12]). It is also worth remarking that $\varepsilon_{i j}^{(N)} \rightarrow 1-\delta_{i j}$ as $N \rightarrow \infty$ for fixed indices $i, j$, so that we may regard the (strict) multiple $L$-values (1.3) as "limiting cases" $S^{(\infty, M)}\left(n_{1}, n_{2}, \ldots, n_{k}\right)$ of our series. We notice that our partial multiple $L$-value 
$S_{k}^{(N, M)}\left(n_{1}, \ldots, n_{k}\right)$ is a special case of neither the multiple $L$-values (1.2) nor (1.3) since $\varepsilon_{i_{1} i_{2} \ldots i_{k}}^{(N)}$ does depend on both the differences $i_{j}-i_{j-1}$ of adjacent indices and the values of the indices $i_{1}, \ldots, i_{k}$ themselves. However, it is not difficult to see that $S_{k}^{(N, M)}\left(n_{1}, \ldots, n_{k}\right)$ can be expressed as a $\mathbb{Q}$-linear combination of (1.2) (or (1.3)). Thus, for fixed $N$ and $M$, it may be interesting to study the structure of the subalgebra spanned by all $S_{k}^{(N, M)}\left(n_{1}, n_{2}, \ldots, n_{k}\right)$ in the $\mathbb{Q}$-algebra spanned by all multiple $L$-values $S_{k}^{(1, M)}\left(n_{1}, \ldots, n_{k}\right)$. We leave these problems to future study.

We now explain the spectral-theoretic origin of our series $S_{k}^{(N, M)}\left(n_{1}, \ldots, n_{k}\right)$. A system of differential equations defined by the operator

$$
Q:=\left(\begin{array}{cc}
\alpha & 0 \\
0 & \beta
\end{array}\right)\left(-\frac{1}{2} \frac{d^{2}}{d x^{2}}+\frac{1}{2} x^{2}\right)+\left(\begin{array}{cc}
0 & -1 \\
1 & 0
\end{array}\right)\left(x \frac{d}{d x}+\frac{1}{2}\right)
$$

having two real parameters $\alpha, \beta$ is called the non-commutative harmonic oscillator. This system was first introduced and extensively studied by Parmeggiani and Wakayama [16, 17] (see also [15]). It is shown that when $\alpha, \beta>0$ and $\alpha \beta>1$, $Q$ defines a positive, self-adjoint operator on $L^{2}(\mathbb{R}) \otimes \mathbb{C}^{2}$ which has only a discrete spectrum $(0<) \lambda_{1} \leq \lambda_{2} \leq \cdots \leq \lambda_{n} \leq \ldots(\nearrow+\infty)$, and the multiplicities of the eigenvalues are uniformly bounded. In order to describe the total behavior of the spectrum, Ichinose and Wakayama [6] studied the spectral zeta function $\zeta_{Q}(s):=\sum_{n=1}^{\infty} \lambda_{n}^{-s}$, which is absolutely convergent if $\Re(s)>1$. This is analytically continued to the whole plane $\mathbb{C}$ and gives a single-valued meromorphic function which has a simple pole at $s=1$ and 'trivial' zeros at non-positive even integers. If $\alpha=\beta=1 / \sqrt{2}$, then $Q$ is unitarily equivalent to a couple of the (ordinary) harmonic oscillators, from which it follows that $\zeta_{Q}(s)=2\left(2^{s}-1\right) \zeta(s)$. Thus one can regard $\zeta_{Q}(s)$ as a deformation of the Riemann zeta function $\zeta(s)$.

In describing the special values of the spectral zeta function $\zeta_{Q}(s)$, the integrals

$$
\begin{aligned}
J_{m}(n)=2^{m} \int_{0}^{1} \ldots \int_{0}^{1}\left(\frac{\left(1-x_{1}^{4}\right)\left(1-x_{2}^{4} \cdots x_{m}^{4}\right)}{\left(1-x_{1}^{2} \cdots x_{m}^{2}\right)^{2}}\right)^{n} \frac{d x_{1} \cdots d x_{m}}{1-x_{1}^{2} \cdots x_{m}^{2}} & \\
& (m=2,3,4, \ldots ; n=0,1,2, \ldots)
\end{aligned}
$$

and their generating functions $g_{m}(x)=\sum_{n=0}^{\infty}\left(\begin{array}{c}-1 / 2 \\ n\end{array}\right) J_{m}(n) x^{n}$ play a very important role. In fact, Ichinose and Wakayama 7] calculated the first two special values $\zeta_{Q}(2)$ and $\zeta_{Q}(3)$ in terms of $g_{2}(x)$ and $g_{3}(x)$, respectively. The higher special values $\zeta_{Q}(m)$ $(m \geq 4)$ are also expected to be expressed by $g_{m}(x)$ and their generalizations (see, e.g., [13, 9, 8]). In the case where $m=2 r$ is even, $J_{2 r}(n)$ is explicitly given by

$$
J_{2 r}(n)=\sum_{p=0}^{n}(-1)^{p}\left(\begin{array}{c}
-\frac{1}{2} \\
p
\end{array}\right)^{2}\left(\begin{array}{l}
n \\
p
\end{array}\right) \sum_{k=0}^{r-1} \zeta\left(2 r-2 k, \frac{1}{2}\right) S_{k, p},
$$

where $\zeta(s, x):=\sum_{n=0}^{\infty}(n+x)^{-s}$ is the Hurwitz zeta function and

$$
S_{k, p}=\sum_{1 \leq i_{1} \leq i_{2} \leq \cdots \leq i_{k} \leq 2 p} \varepsilon_{i_{1} i_{2} \ldots i_{k}}^{(2)} \frac{(-1)^{i_{1}+i_{2}+\cdots+i_{k}}}{i_{1}^{2} i_{2}^{2} \ldots i_{k}^{2}} .
$$

Now it is immediate to see that our series $S_{k}^{(N, M)}\left(n_{1}, \ldots, n_{k}\right)$ is a natural generalization of $S_{k}^{(2)}(2)=\lim _{p \rightarrow \infty} S_{k, p}$ (we give the explicit formula of $S_{k}^{(2)}(2)$ in Example 3.4). 
It is also worth remarking that another kind of generating function $w_{2}(t)=$ $\sum_{n=0}^{\infty} J_{2}(n) t^{n}$ of $J_{2}(n)$ is regarded as a period integral for the universal family of the elliptic curves equipped with a rational point of order 4 and that it satisfies a Picard-Fuchs differential equation attached to this family of curves [10].

Conventions. We recall several basic conventions on partitions and symmetric functions (for further details, see $[\underline{5}$ ).

A partition is a weakly decreasing sequence of non-negative integers which has finitely many non-zero entries. For a partition $\lambda=\left(\lambda_{1}, \ldots, \lambda_{l}\right)\left(\lambda_{l} \geq 1\right)$, the sum $\lambda_{1}+\cdots+\lambda_{l}$ of entries in $\lambda$ is denoted by $|\lambda|$ and the number $l$ of non-zero entries in $\lambda$ is denoted by $\ell(\lambda)$. We write $\lambda \vdash k$ to imply $|\lambda|=k$, and say $\lambda$ is a partition of $k$. We denote by $\emptyset$ the (only) partition of 0 . To indicate a multiple of the same numbers in $\lambda$, we often write in an exponential form; Let $m_{i}=m_{i}(\lambda)$ be the number of $i$ 's in $\lambda$. We call $m_{i}(\lambda)$ the multiplicity of $i$ in $\lambda$. Then, we also write $\lambda=\left(k^{m_{k}}, \ldots, 2^{m_{2}}, 1^{m_{1}}\right)$ or $\lambda=1^{m_{1}} 2^{m_{2}} \ldots k^{m_{k}}$. For instance, $\lambda=(4,2,2,1,1,1)$ is also written as $\lambda=\left(4,2^{2}, 1^{3}\right)=1^{3} 2^{2} 4^{1}$. When all the entries of $\lambda$ are even, we call $\lambda$ an even partition. For a given partition $\mu=\left(\mu_{1}, \ldots, \mu_{l}\right)$ and a positive integer $q$, we define $q \mu=\left(q \mu_{1}, \ldots, q \mu_{l}\right)$. We notice that $\{\lambda \vdash 2 k \mid \lambda$ : even $\}=\{2 \mu \mid \mu \vdash k\}$. If a given pair of two partitions $\lambda$ and $\mu$ satisfies that $\lambda_{i}-\mu_{i}=0$ or 1 for any index $i$, then we say $\lambda / \mu$ is a vertical strip. For instance, $(4,2,2,1,1,1) /(3,2,1,1)$ is a vertical strip.

Let $f(n)$ be a function on $\mathbb{N}$ and $a_{n}$ a sequence. Then, for a partition $\lambda$ and $q \in \mathbb{N}$, we put $f(q \lambda):=\prod_{j=1}^{\ell(\lambda)} f\left(q \lambda_{j}\right)$ and $a_{q \lambda}:=\prod_{j=1}^{\ell(\lambda)} a_{q \lambda_{j}}$. For instance, $(q \lambda) !=$ $\prod_{j=1}^{\ell(\lambda)}\left(q \lambda_{j}\right)$ !.

Let $x_{1}, x_{2}, \ldots$ be (infinitely many) variables. For each positive integer $r$, we respectively denote by $e_{r}=e_{r}\left(x_{1}, x_{2}, \ldots\right)$ and $h_{r}=h_{r}\left(x_{1}, x_{2}, \ldots\right)$ the $r$-th elementary and $r$-th complete symmetric function defined by

$$
e_{r}=\sum_{1 \leq i_{1}<i_{2}<\cdots<i_{r}} x_{i_{1}} x_{i_{2}} \ldots x_{i_{r}}, \quad h_{r}=\sum_{1 \leq i_{1} \leq i_{2} \leq \cdots \leq i_{r}} x_{i_{1}} x_{i_{2}} \ldots x_{i_{r}} .
$$

We also put $e_{0}=h_{0}=1$ for convenience. Moreover, for a partition $\lambda$, we put $e_{\lambda}=\prod_{i \geq 1} e_{\lambda_{i}}$ and $h_{\lambda}=\prod_{i \geq 1} h_{\lambda_{i}}$. The generating functions of $e_{r}$ and $h_{r}$ are given by

$$
E(t)=\sum_{r=0}^{\infty} e_{r} t^{r}=\prod_{n=1}^{\infty}\left(1+x_{n} t\right), \quad H(t)=\sum_{r=0}^{\infty} h_{r} t^{r}=\prod_{n=1}^{\infty}\left(1-x_{n} t\right)^{-1}
$$

\section{Generating functions}

In this section, we establish generating function formulas for the series $S_{k}^{(N, M)}(n)$. To achieve this, we first consider a decomposition of the non-strict multiple sum $S_{k}^{(N, M)}(n)$ into the sum of several strict multiple sums. Notice that each increasing sequence $1 \leq i_{1} \leq i_{2} \leq \cdots \leq i_{k}$ of $k$ positive integers uniquely determines a sequence $\boldsymbol{r}=\left(r_{1}, r_{2}, \ldots, r_{l}\right)$, which we will refer to as the multiplicity of the sequence 
$\left(i_{1}, i_{2}, \ldots, i_{k}\right)$, through the condition

$$
\begin{aligned}
\underbrace{i_{1}=\cdots=i_{r_{1}}}_{r_{1}}<\underbrace{i_{r_{1}+1}=\cdots=i_{r_{1}+r_{2}}}_{r_{2}}<i_{r_{1}+r_{2}+1}=\ldots \\
=i_{r_{1}+\cdots+r_{l-1}}<\underbrace{i_{r_{1}+\cdots+r_{l-1}+1}=\cdots=i_{r_{1}+\cdots+r_{l}}}_{r_{l}} .
\end{aligned}
$$

Obviously, $\boldsymbol{r}$ is a permutation of a certain partition of $k$. We denote by $\widetilde{S}^{(N, M)}(n ; \boldsymbol{r})$ the partial sum of $S^{(N, M)}(n)$ whose running indices have multiplicity $\boldsymbol{r}$, i.e.

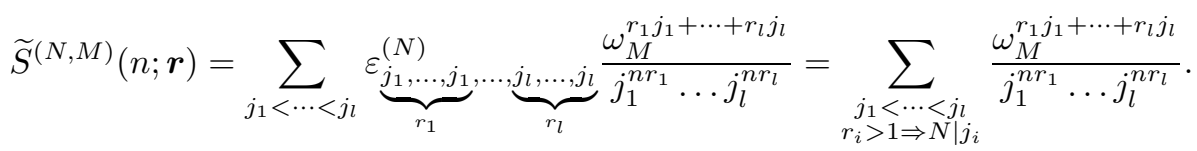

We also put

$$
S^{(N, M)}(n ; \emptyset)=1, \quad S^{(N, M)}(n ; \lambda)=\sum_{r \in P(\lambda)} \widetilde{S}^{(N, M)}(n ; \boldsymbol{r}) \quad(\lambda \neq \emptyset),
$$

where $P(\lambda)$ denotes the set consisting of the permutations of a partition $\lambda$. It is easy to see that

$$
S_{k}^{(N, M)}(n)=\sum_{\lambda \vdash k} S^{(N, M)}(n ; \lambda) .
$$

To study the series $S^{(N, M)}(n ; \lambda)$, we here employ another function $R^{(N, M)}(n ; \mu)$ defined by

$R^{(N, M)}(n ; \emptyset):=1, \quad R^{(N, M)}(n ; \mu):=S^{(N, M)}\left(n ; \mu_{>1}\right) S^{(N, M)}\left(n ; 1^{m_{1}(\mu)}\right) \quad(\mu \neq \emptyset)$.

Here $\mu_{>1}$ denotes the partition defined by $\mu_{>1}:=2^{m_{2}(\mu)} 3^{m_{3}(\mu)} \ldots$ Fix a partition $\mu \vdash k$ and put $q=m_{1}(\mu), p=\ell(\mu)-q$. We easily see that

$$
\begin{aligned}
& R^{(N, M)}(n ; \mu)=\sum_{\boldsymbol{r} \in P\left(\mu_{>1}\right)} \widetilde{R}_{p, q}^{(N, M)}(n ; \boldsymbol{r}), \\
& \widetilde{R}_{p, q}^{(N, M)}(n ; \boldsymbol{r})=\sum_{\substack{s_{1}<\cdots<s_{p} \\
t_{1}<\cdots<t_{q} \\
N \mid s_{i}}} \frac{\omega_{M}^{r_{1} s_{1}+\cdots+r_{p} s_{p}+t_{1}+\cdots+t_{q}}}{s_{1}^{r_{1} n} \ldots s_{p}^{r_{p} n} t_{1}^{n} \ldots t_{q}^{n}} .
\end{aligned}
$$

In the sum $\widetilde{R}_{p, q}^{(N, M)}(n ; \boldsymbol{r})$ for each $\boldsymbol{r} \in P\left(\mu_{>1}\right)$, several of the running indices $t_{1}, \ldots, t_{q}$ may coincide with certain $s_{1}, \ldots, s_{p}$. To describe the situation, we introduce the following map: Put

$$
\begin{aligned}
& I(p, q)= \\
& \left\{(\boldsymbol{\tau}, \boldsymbol{\varepsilon})=\left(\tau_{0}, \tau_{1}, \ldots, \tau_{p}, \varepsilon_{1}, \ldots, \varepsilon_{p}\right) \mid \tau_{i} \in \mathbb{Z}_{\geq 0}, \varepsilon_{i} \in\{0,1\}, \sum_{i=0}^{p} \tau_{i}+\sum_{i=1}^{p} \varepsilon_{i}=q\right\} .
\end{aligned}
$$

For each element $(\boldsymbol{r},(\boldsymbol{\tau}, \boldsymbol{\varepsilon})) \in P\left(\mu_{>1}\right) \times I(p, q)$, we associate a new sequence $\pi_{\mu}(\boldsymbol{r},(\boldsymbol{\tau}, \boldsymbol{\varepsilon}))$ by

$$
\pi_{\mu}(\boldsymbol{r},(\boldsymbol{\tau}, \boldsymbol{\varepsilon}))=\left(1^{\tau_{0}}, r_{1}+\varepsilon_{1}, 1^{\tau_{1}}, r_{2}+\varepsilon_{2}, \ldots, r_{p}+\varepsilon_{p}, 1^{\tau_{p}}\right) .
$$


Notice that there exists a partition $\lambda \vdash k$ such that $\lambda / \mu_{>1}$ is a vertical strip and $\pi_{\mu}(\boldsymbol{r},(\boldsymbol{\tau}, \varepsilon)) \in P(\lambda)$. Namely, the correspondence $\pi_{\mu}$ defines a map $\pi_{\mu}: P\left(\mu_{>1}\right) \times$

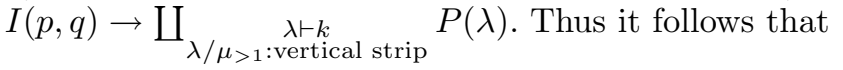

$$
\sum_{\boldsymbol{r} \in P(\mu>1)} \widetilde{R}_{p, q}^{(N, M)}(n ; \boldsymbol{r})=\sum_{\substack{\lambda \vdash k \\ \lambda / \mu>1 \text { vertical strip }}} \sum_{\boldsymbol{r} \in P(\lambda)}\left|\pi_{\mu}^{-1}(\boldsymbol{r})\right| \widetilde{S}^{(N, M)}(n ; \boldsymbol{r}) .
$$

Since each $\left|\pi_{\mu}^{-1}(\boldsymbol{r})\right|$ depends only on $\lambda$, we obtain

$$
R^{(N, M)}(n ; \mu)=\sum_{\substack{\lambda \vdash k \\ \lambda / \mu>1 \text { vertical strip }}}\left|\pi_{\mu}^{-1}(\lambda)\right| S^{(N, M)}(n ; \lambda) .
$$

Next, we calculate $\left|\pi_{\mu}^{-1}(\lambda)\right|$. For each $a>2$, we assume that $\lambda_{i_{a 1}}=\cdots=\lambda_{i_{a, d(a)}}=$ $a$, where $d(a)=m_{a}(\lambda)$. Let us count the number of elements $(\boldsymbol{r},(\boldsymbol{\tau}, \boldsymbol{\varepsilon}))$ in $I(p, q)$ such that $\pi_{\mu}(\boldsymbol{r},(\boldsymbol{\tau}, \boldsymbol{\varepsilon}))=\lambda$. Notice that $\boldsymbol{\tau}$ is uniquely determined by the assumption. If $r_{i_{a j}}+\varepsilon_{i_{a j}}=\lambda_{i_{a j}}=a$, then it is possible that $\left(r_{i_{a j}}, \varepsilon_{i_{a j}}\right)=(a, 0)$ or $(a-1,1)$, and there are exactly $\left(\begin{array}{c}m_{a}(\lambda) \\ m_{a}(\lambda ; \mu)\end{array}\right)$ ways of choosing $i_{a j}$ such that $\left(r_{i_{a j}}, \varepsilon_{i_{a j}}\right)=(a, 0)$, where $m_{i}(\lambda ; \mu)=\left|\left\{j \mid \lambda_{j}=\mu_{j}=i\right\}\right|$. (Remark that $m_{2}(\lambda ; \mu)=m_{2}(\lambda)$.) Thus we have $\left|\pi_{\mu}^{-1}(\lambda)\right|=\prod_{i>2}\left(\begin{array}{c}m_{a}(\lambda) \\ m_{a}(\lambda ; \mu)\end{array}\right)$. If $\mu$ is an even partition and $\mu / \lambda_{>1}$ is a vertical strip, then $m_{i}(\lambda ; \mu)=m_{i}(\lambda)$ (if $i$ is even) or 0 (if $i$ is odd) by definition, and hence $\left|\pi_{\mu}^{-1}(\lambda)\right|=1$. Consequently, we get the following lemma.

Lemma 2.1. For each $\mu \vdash k$, it follows that

$$
R^{(N, M)}(n ; \mu)=\sum_{\substack{\lambda \vdash k \\
\lambda / \mu>1}} \prod_{i>2}\left(\begin{array}{c}
m_{i}(\lambda) \\
m_{i}(\lambda ; \mu)
\end{array}\right) S^{(N, M)}(n ; \lambda),
$$

where $m_{i}(\lambda ; \mu)=\left|\left\{j \mid \lambda_{j}=\mu_{j}=i\right\}\right|$. In particular, if $\mu$ is even, then

$$
R^{(N, M)}(n ; \mu)=\sum_{\substack{\lambda \vdash k \\ \lambda / \mu>1 \text { :vertical strip }}} S^{(N, M)}(n ; \lambda) .
$$

Lemma 2.2. For any $\lambda \vdash k$, there uniquely exists $\mu \vdash k$ such that $\mu_{>1}$ is even and $\lambda / \mu_{>1}$ is a vertical strip.

Proof. It is immediate to see that

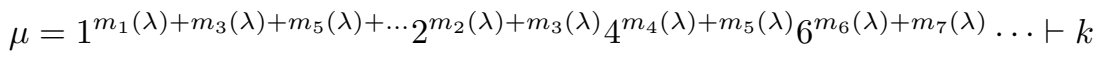

is the unique partition which satisfies all the desired conditions.

By Lemmas 2.1 and 2.2, we readily obtain the

Lemma 2.3. Let $U_{d}^{(N, M)}(n):=\sum_{\mu \vdash d} S^{(N, M)}(n ; 2 \mu)$. Then it follows that

$$
\begin{aligned}
S_{k}^{(N, M)}(n) & =\sum_{\lambda \vdash k} S^{(N, M)}(n ; \lambda)=\sum_{\substack{\mu \vdash k \\
\mu>1}} R^{(N, M)}(n ; \mu) \\
& =\sum_{0 \leq 2 d \leq k} S^{(N, M)}\left(n ; 1^{k-2 d}\right) U_{d}^{(N, M)}(n) .
\end{aligned}
$$


We next study the generating function of $S_{k}^{(N, M)}(n)$. For this purpose, the following formula, which is obtained by the canonical product expression of the gamma function, is useful.

Lemma 2.4. For $a_{i}, b_{i} \in \mathbb{C}$ satisfying $\sum_{i=1}^{l} a_{i}=\sum_{i=1}^{l} b_{i}$, the equality

$$
\prod_{m=k}^{\infty} \prod_{j=1}^{l} \frac{m+a_{j}}{m+b_{j}}=\prod_{j=1}^{l} \frac{\Gamma\left(k+b_{j}\right)}{\Gamma\left(k+a_{j}\right)}
$$

holds for any integer $k$.

Lemma 2.5. The generating function of $U_{d}^{(N, M)}(n)$ is given by

$$
\mathcal{H}^{(N, M)}(n ; x):=\sum_{d=0}^{\infty} U_{d}^{(N, M)}(n) x^{2 n d}=\prod_{k=1}^{M} \prod_{j=0}^{2 n-1} \frac{\Gamma\left(\frac{1}{M}\left(k-\frac{1}{N} \omega_{2 n}^{j} \omega_{M n}^{k N} x\right)\right)}{\Gamma\left(\frac{k}{M}\right)}
$$

Proof. We notice that

$$
U_{d}^{(N, M)}(n)=\sum_{\mu \vdash d} S^{(N)}(n ; 2 \mu)=h_{d}\left(\frac{\omega_{M}^{2 N}}{N^{2 n}}, \frac{\omega_{M}^{4 N}}{(2 N)^{2 n}}, \frac{\omega_{M}^{6 N}}{(3 N)^{2 n}}, \ldots\right)
$$

since the complete symmetric function $h_{d}$ is the sum of all monomials of degree $d$. Therefore, by specializing $x_{m}=\omega_{M}^{2 m N} /(N m)^{2 n}$ and $t=x^{2 n}$ in the generating function $H(t)$ in (1.6), we obtain

$$
\begin{aligned}
\mathcal{H}^{(N, M)}(n ; x) & =\prod_{m=1}^{\infty}\left(1-\frac{\omega_{M}^{2 m N}}{(N m)^{2 n}} x^{2 n}\right)^{-1}=\prod_{m=0}^{\infty} \prod_{k=1}^{M}\left\{1-\left(\frac{\omega_{M n}^{k N} x}{N(M m+k)}\right)^{2 n}\right\}^{-1} \\
& =\prod_{m=0}^{\infty} \prod_{k=1}^{M} \prod_{j=0}^{2 n-1}\left(1-\omega_{2 n}^{j} \frac{\omega_{M n}^{k N} x}{N(M m+k)}\right)^{-1} \\
& =\prod_{m=0}^{\infty} \prod_{k=1}^{M} \prod_{j=0}^{2 n-1} \frac{m+\frac{k}{M}-\frac{\omega_{2 n}^{j} \omega_{M n}^{k N} x}{M N}}{m+\frac{k}{M}}
\end{aligned}
$$

Applying Lemma 2.4 to the equation above, we have (2.6).

Lemma 2.6. The generating function of $S^{(N, M)}\left(n ; 1^{r}\right)$ is given by

$$
\mathcal{E}^{(M)}(n ; x):=\sum_{r=0}^{\infty} S^{(N, M)}\left(n ; 1^{r}\right) x^{n r}=\prod_{k=1}^{M} \prod_{j=0}^{n-1} \frac{\Gamma\left(\frac{k}{M}\right)}{\Gamma\left(\frac{1}{M}\left(k-\omega_{2 n}^{2 j-1} \omega_{M n}^{k} x\right)\right)}
$$

Proof. We notice that

$$
S^{(N)}\left(n ; 1^{r}\right)=e_{r}\left(\frac{\omega_{M}}{1^{n}}, \frac{\omega_{M}^{2}}{2^{n}}, \frac{\omega_{M}^{3}}{3^{n}}, \ldots\right)
$$

Hence, if we specialize $x_{m}=\omega_{M}^{m} / m^{n}$ and set $t=x^{n}$ in the generating function $E(t)$ in (1.6), then we obtain the lemma by a similar calculation as in the case of $\mathcal{H}^{(N, M)}(n ; x)$. 
Now, we obtain the following.

Theorem 2.7. The generating function of $S_{k}^{(N, M)}(n)$ is given by $(2.8)$

$$
\mathcal{S}^{(N, M)}(n ; x):=\sum_{k=0}^{\infty} S_{k}^{(N)}(n) x^{n k}=\prod_{k=1}^{M} \frac{\prod_{j=0}^{2 n-1} \Gamma\left(\frac{1}{M}\left(k-\frac{1}{N} \omega_{2 n}^{j} \omega_{M n}^{k N} x\right)\right)}{\Gamma\left(\frac{k}{M}\right)^{n} \prod_{j=0}^{n-1} \Gamma\left(\frac{1}{M}\left(k-\omega_{2 n}^{2 j-1} \omega_{M n}^{k} x\right)\right)} .
$$

Proof. It is clear that $\mathcal{S}^{(N, M)}(n ; x)=\mathcal{H}^{(N, M)}(n ; x) \mathcal{E}^{(M)}(n ; x)$ from equation (2.4). Hence one immediately obtains the formula (2.8) from (2.6) and (2.7).

If $M \mid N$, then, using the Gauss-Legendre formula of the gamma function, we have the following reduced formulas:

$$
\begin{aligned}
& \mathcal{H}^{(N, M)}(n ; x)=\prod_{j=0}^{2 n-1} \Gamma\left(1-\frac{\omega_{2 n}^{j} x}{N}\right), \\
& \mathcal{S}^{(N, M)}(n ; x)=\prod_{k=1}^{M} \frac{\Gamma\left(\frac{k}{M}\right)^{n} \prod_{j=0}^{2 n-1} \Gamma\left(1-\frac{\omega_{2 n}^{j} x}{N}\right)}{\prod_{j=0}^{n-1} \Gamma\left(\frac{1}{M}\left(k-\omega_{2 n}^{2 j-1} \omega_{M n}^{k} x\right)\right)} .
\end{aligned}
$$

Notice that $\mathcal{E}^{(M)}(n ; x)$ depends only on $M$.

\section{Partial alternating multiple zeta Values}

In this section, we concentrate on the special case where $N=M=2$. From the definition, the sums $S_{k}(n):=S_{k}^{(2,2)}(n)$ in this case may be called partial alternating multiple zeta values. From (2.10), we have

$$
\begin{aligned}
\mathcal{S}(n ; x):=\mathcal{S}^{(2)}(n ; x) & =\frac{\Gamma\left(\frac{1}{2}\right)^{n} \prod_{j=0}^{2 n-1} \Gamma\left(1-\frac{x}{2} \omega_{2 n}^{j}\right)}{\prod_{j=0}^{n-1} \Gamma\left(\frac{1}{2}-\frac{x}{2} \omega_{n}^{j}\right) \Gamma\left(1-\frac{x}{2} \omega_{n}^{j} \omega_{2 n}\right)} \\
& =\frac{\Gamma\left(\frac{1}{2}\right)^{n} \prod_{j=0}^{n-1} \Gamma\left(1-\frac{x}{2} \omega_{n}^{j}\right)}{\prod_{j=0}^{n-1} \Gamma\left(\frac{1}{2}-\frac{x}{2} \omega_{n}^{j}\right)} .
\end{aligned}
$$

Furthermore, using the duplication formula $\Gamma(2 a) \Gamma(1 / 2)=2^{2 a-1} \Gamma(a) \Gamma(1 / 2+a)$ with $a=-x \omega_{n}^{j} / 2$ and the equation $\sum_{j=0}^{n-1} \omega_{n}^{j}=\delta_{n, 1}$, we see that

$$
\mathcal{S}(n ; x)=\frac{\Gamma\left(\frac{1}{2}\right)^{n} \prod_{j=0}^{n-1} \Gamma\left(1-\frac{x}{2} \omega_{n}^{j}\right)}{\prod_{j=0}^{n-1} \Gamma\left(-x \omega_{n}^{j}\right) \Gamma\left(\frac{1}{2}\right) 2^{x \omega_{n}^{j}+1} \Gamma\left(-\frac{x}{2} \omega_{n}^{j}\right)^{-1}}=2^{-x \delta_{n, 1}} \prod_{j=0}^{n-1} \frac{\Gamma\left(1-\frac{x}{2} \omega_{n}^{j}\right)^{2}}{\Gamma\left(1-x \omega_{n}^{j}\right)} .
$$

For $m \geq 0$, define the sequence $\left\{A^{\bullet}(m)\right\}_{m \geq 0}$ by $A^{\bullet}(0):=1, A^{\bullet}(1):=0$ and

$$
A^{\bullet}(m):=\sum_{a=1}^{m-1} \zeta_{a}^{\bullet}(\underbrace{1, \ldots, 1}_{a-1}, m-a+1) \quad(m \geq 2) .
$$

Namely, $A^{\bullet}(m)(m \geq 2)$ denotes the sum of multiple zeta values of weight $m$ and height 1. It is known that $A^{\bullet}(m)$ can be expressed as a polynomial in $\zeta(2), \zeta(3), \ldots$ 
and $\zeta(m)$ with rational coefficients (see [14]). For example, we have $A^{\bullet}(3)=\zeta(3)+$ $\zeta_{2}^{\bullet}(1,2)=2 \zeta(3)$ since $\zeta_{2}^{\bullet}(1,2)=\zeta(3)$, which is due to Euler. Furthermore, we put

$$
A_{n}^{\bullet}(m):=\sum_{\substack{m_{1}, \ldots, m_{n} \geq 0 \\ m_{1}+\cdots+m_{n}=m}} A^{\bullet}\left(m_{1}\right) \cdots A^{\bullet}\left(m_{n}\right), \quad Z_{n}(k):=\sum_{\substack{\mu \vdash k \\ \mu_{\ell(\mu)}>\delta_{n, 1}}} \frac{\nu(n \mu)}{z_{\mu}} \zeta(n \mu),
$$

where $\nu(x):=2^{1-x}-1$ and $z_{\mu}:=\prod_{i \geq 1} i^{m_{i}(\mu)} m_{i}(\mu)$ !. Note that $A_{1}^{\bullet}(m)=A^{\bullet}(m)$. Then, we get the following expressions of the values $S_{k}(n)=S_{k}^{(2)}(n)$.

Theorem 3.1. (i) If $n=1$, then

$$
\begin{aligned}
S_{k}(1) & =\sum_{m=0}^{k} \frac{(-\log 2)^{k-m}}{(k-m) ! 2^{m}} A^{\bullet}(m) \\
& =\sum_{m=0}^{k} \frac{(-\log 2)^{k-m}}{(k-m) !} Z_{1}(m) \in \mathbb{Q}[\log 2, \zeta(2), \zeta(3), \ldots, \zeta(k)] .
\end{aligned}
$$

(ii) If $n \geq 2$, then

$$
S_{k}(n)=\frac{1}{2^{n k}} A_{n}^{\bullet}(n k)=Z_{n}(k) \in \mathbb{Q}[\zeta(n), \zeta(2 n), \ldots, \zeta(k n)] .
$$

Proof. From the generating function (3.1), it is sufficient to show that

$$
\prod_{j=0}^{n-1} \frac{\Gamma\left(1-\frac{x}{2} \omega_{n}^{j}\right)^{2}}{\Gamma\left(1-x \omega_{n}^{j}\right)}=\sum_{m=0}^{\infty} A_{n}^{\bullet}(n m)\left(\frac{x}{2}\right)^{n m}=\sum_{m=0}^{\infty} Z_{n}(m) x^{n m}
$$

To prove this, we recall the identity (see $[1,4]$ )

$$
\begin{aligned}
\frac{\Gamma(1-X) \Gamma(1-Y)}{\Gamma(1-X-Y)} & =1-\sum_{a, b=1}^{\infty} \zeta_{a}^{\bullet}(\underbrace{1, \ldots, 1}_{a-1}, b+1) X^{a} Y^{b} \\
& =\exp \left(\sum_{m=2}^{\infty} \frac{X^{m}+Y^{m}-(X+Y)^{m}}{m} \zeta(m)\right) .
\end{aligned}
$$

Putting $X=Y=x \omega_{n}^{j} / 2$ and writing $a+b=m$ in the middle term in (3.5), we have

$$
\frac{\Gamma\left(1-\frac{x}{2} \omega_{n}^{j}\right)^{2}}{\Gamma\left(1-x \omega_{n}^{j}\right)}=\sum_{m=0}^{\infty} A^{\bullet}(m)\left(\frac{x \omega_{n}^{j}}{2}\right)^{m}=\exp \left(\sum_{m=2}^{\infty} \frac{\nu(m)}{m} \zeta(m)\left(\omega_{n}^{j} x\right)^{m}\right) .
$$

Then, taking the product $\prod_{j=0}^{n-1}$ of this equation, one sees that

$$
\prod_{j=0}^{n-1} \frac{\Gamma\left(1-\frac{x}{2} \omega_{n}^{j}\right)^{2}}{\Gamma\left(1-x \omega_{n}^{j}\right)}=\sum_{m=0}^{\infty} A_{n}^{\bullet}(m)\left(\frac{x \omega_{n}^{j}}{2}\right)^{m}=\exp \left(\sum_{\substack{m=1 \\ n m \geq 2}}^{\infty} \frac{\nu(n m)}{m} \zeta(n m) x^{n m}\right)
$$


because $\sum_{j=0}^{n-1} \omega_{n}^{j m}=n$ if $n \mid m$ and 0 otherwise. Here, the rightmost side of (3.6) can be written as

$$
\begin{aligned}
& \prod_{\substack{m=1 \\
n m \geq 2}}^{\infty} \exp \left(\frac{\nu(n m)}{m} \zeta(n m) x^{n m}\right) \\
= & \prod_{\substack{m=1 \\
n m \geq 2}}^{\infty} \sum_{l_{m}=0}^{\infty} \frac{1}{l_{m} !}\left(\frac{\nu(n m)}{m} \zeta(n m) x^{n m}\right)^{l_{m}} \\
= & \begin{cases}\sum_{l_{2}, l_{3}, \ldots=0}^{\infty} \frac{\nu(2)^{l_{2}} \nu(3)^{l_{3}} \cdots}{\left(2^{l_{2}} 3^{l_{3}} \cdots\right)\left(l_{2} ! l_{3} ! \cdots\right)}\left(\zeta(2)^{l_{1}} \zeta(3)^{l_{3}} \cdots\right) x^{2 l_{2}+3 l_{3}+\cdots} \quad(n=1) \\
\sum_{l_{1}, l_{2}, \ldots=0}^{\infty} \frac{\nu(n)^{l_{1}} \nu(2 n)^{l_{2}} \cdots}{\left(1^{l_{1}} 2^{l_{2}} \cdots\right)\left(l_{1} ! l_{2} ! \cdots\right)}\left(\zeta(n)^{l_{1}} \zeta(2 n)^{l_{2}} \cdots\right) x^{l_{1}+2 l_{2}+\cdots} & (n \geq 2) \\
= & \sum_{m=0}^{\infty}\left\{\sum_{\substack{\mu \vdash m \\
\mu_{\ell}(\mu)>\delta_{n, 1}}}^{\infty} \frac{\nu(n \mu)}{z_{\mu}} \zeta(n \mu)\right\} x^{n m}=\sum_{m=0}^{\infty} Z_{n}(m) x^{n m} .\end{cases}
\end{aligned}
$$

Note that from the second equality in (3.6), this shows that $A_{n}^{\bullet}(m)=0$ if $n \nmid m$. Therefore, one can actually obtain the equations (3.4). This completes the proof of the theorem.

Example 3.2. We have

$$
\begin{aligned}
& S_{1}(1)=-\log 2, \quad S_{2}(1)=\frac{(\log 2)^{2}}{2}-\frac{\zeta(2)}{4}, \\
& S_{3}(1)=-\frac{(\log 2)^{3}}{6}+\frac{\log 2}{4} \zeta(2)-\frac{1}{4} \zeta(3),
\end{aligned}
$$

and

$$
\begin{aligned}
& S_{1}(3)=-\frac{3}{4} \zeta(3), \quad S_{2}(3)=-\frac{31}{64} \zeta(6)+\frac{9}{32} \zeta(3)^{2} \\
& S_{3}(3)=-\frac{255}{768} \zeta(9)+\frac{93}{128} \zeta(6) \zeta(3)-\frac{27}{384} \zeta(3)^{3} .
\end{aligned}
$$

If one further assumes that $n$ is even, then one can obtain the following various expressions.

Theorem 3.3. It follows that

$$
\begin{aligned}
S_{k}(2 n) & =\left(-\pi^{2}\right)^{n k} \sum_{\substack{m_{1}, \ldots, m_{n} \geq 0 \\
m_{1}+\cdots+m_{n}=n k}} \omega_{n}^{m_{1}+2 m_{2}+\cdots+n m_{n}} \frac{B_{2 m_{1}}}{\left(2 m_{1}\right) !} \cdots \frac{B_{2 m_{n}}}{\left(2 m_{n}\right) !} \\
& =\left(-\pi^{2}\right)^{n k} \sum_{\substack{\lambda \vdash n k \\
\ell(\lambda) \leq n}}\left\langle p_{n} \circ h_{k}, m_{\lambda}\right\rangle \frac{B_{2 \lambda}}{(2 \lambda) !} \\
& =\left(-\pi^{2}\right)^{n k} \sum_{\mu \vdash k} \frac{\widetilde{\nu}(2 n \mu)}{z_{\mu}} \frac{B_{2 n \mu}}{(2 n \mu) !},
\end{aligned}
$$

where $\widetilde{\nu}(x):=2^{x-1}-1, p_{n}$ is the $n$-th power-sum symmetric function, $m_{\lambda}$ is the monomial symmetric function for $\lambda$, ○ is the plethysm, and $\langle\cdot, \cdot\rangle$ is the standard 
scalar product in the ring of symmetric functions defined by $\left\langle h_{\lambda}, m_{\mu}\right\rangle=\delta_{\lambda \mu}$ with $\delta_{\lambda \mu}$ being the Kronecker delta (see [5] for details).

Proof. If we apply the reflection formula for the gamma function in (3.1), then we have

$$
\begin{aligned}
\mathcal{S}(2 n ; x) & =\prod_{j=1}^{n} \frac{\Gamma\left(1-\frac{\omega_{2 n}^{j} x}{2}\right)^{2} \Gamma\left(1+\frac{\omega_{2 n}^{j} x}{2}\right)^{2}}{\Gamma\left(1-\omega_{2 n}^{j} x\right) \Gamma\left(1+\omega_{2 n}^{j} x\right)} \\
& =\prod_{j=1}^{n} \frac{\pi x \omega_{2 n}^{j}}{2} \cot \frac{\omega_{2 n}^{j} \pi x}{2}=\prod_{j=1}^{n} \sum_{m=0}^{\infty} \frac{\left(-\omega_{n}^{j}\right)^{m} B_{2 m} \pi^{2 m}}{(2 m) !} x^{2 m},
\end{aligned}
$$

from which we immediately obtain (3.7). Next, it readily follows from (3.7) that

$$
S_{k}^{(2 n)}=\left(-\pi^{2}\right)^{n k} \sum_{\substack{\lambda \vdash n k \\ \ell(\lambda) \leq n}} m_{\lambda}\left(1, \omega_{n}, \ldots, \omega_{n}^{n-1}, 0, \ldots\right) \frac{B_{2 \lambda}}{(2 \lambda) !} .
$$

Thus we should calculate $m_{\lambda}\left(1, \omega_{n}, \ldots, \omega_{n}^{n-1}, 0, \ldots\right)$. Let us recall the expansion formula (see, e.g., [5])

$$
\prod_{i, j \geq 1} \frac{1}{1-x_{i} y_{j}}=\sum_{\lambda} h_{\lambda}(x) m_{\lambda}(y) .
$$

If we set $y_{j}=\omega_{n}^{j-1}$ for $j=1,2, \ldots, n$ and $y_{j}=0$ for $j>n$ in (3.11), then we have

$$
\begin{aligned}
& \sum_{\ell(\lambda) \leq n} h_{\lambda}(x) m_{\lambda}\left(1, \omega_{n}, \ldots, \omega_{n}^{n-1}, 0, \ldots\right) \\
= & \prod_{i \geq 1} \frac{1}{1-x_{i}^{n}}=\sum_{k=0}^{\infty} h_{k}\left(x_{1}^{n}, x_{2}^{n}, \ldots\right)=\sum_{k=0}^{\infty} p_{n} \circ h_{k} .
\end{aligned}
$$

By taking the terms of homogeneous degree $n k$ in (3.12), we have

$$
\sum_{\substack{\lambda \vdash n k \\ \ell(\lambda) \leq n}} h_{\lambda} m_{\lambda}\left(1, \omega_{n}, \ldots, \omega_{n}^{n-1}, 0, \ldots\right)=p_{n} \circ h_{k}
$$

for each $k$. Hence we get $m_{\lambda}\left(1, \omega_{n}, \ldots, \omega_{n}^{n-1}, 0, \ldots\right)=\left\langle p_{n} \circ h_{k}, m_{\lambda}\right\rangle$, which readily implies (3.8). The equation (3.9) follows immediately from (3.3) together with the classical result $\zeta(2 m)=(-1)^{m-1} 2^{2 m-1} B_{2 m} \pi^{2 m} /(2 m)$ ! due to Euler. This completes the proof.

Example 3.4. From the equation (3.7), we have

$$
S_{k}(2)=\frac{(-1)^{k} B_{2 k}}{(2 k) !} \pi^{2 k}=-\frac{\zeta(2 k)}{2^{2 k-1}}, \quad S_{k}(4)=\left\{\sum_{m=0}^{2 k}(-1)^{m} \frac{B_{2 m} B_{4 k-2 m}}{(2 m) !(4 k-2 m) !}\right\} \pi^{4 k} .
$$

See [19] for a similar discussion on the multiple Dirichlet $L$-values.

Remark 3.5. It is remarkable that $S_{k}(2)=S_{k}^{(2)}(2)$ can be reduced as above. We recall that $S_{k}^{(2)}(2)$ is closely related to the special value $\zeta_{Q}(2)$ of the spectral zeta function. Can one explain the simplicity (or "exact solvability") of $S_{k}^{(2)}(2)$ by, for instance, the existence of the Picard-Fuchs differential equation for $w_{2}(t)$ ? 
Remark 3.6. Let us give an example of the partial alternating double zeta value with distinct indices:

$$
\begin{aligned}
S_{2}^{(2)}(1,2 k)= & (k+1) S_{1}^{(2)}(2 k+1)+2\left(1-2^{-2 k}\right) \zeta(2 k) \log 2 \\
& -\sum_{p=1}^{k-1} S_{1}^{(2)}(2 p+1) \zeta(2 k-2 p) \\
S_{2}^{(2)}(2 k, 1)= & -k S_{1}^{(2)}(2 k+1)-\zeta(2 k) \log 2 \\
& +\sum_{p=1}^{k-1} S_{1}^{(2)}(2 p+1) \zeta(2 k-2 p) .
\end{aligned}
$$

Notice that $S_{1}^{(2)}(n)=\left(2^{1-n}-1\right) \zeta(n)$ for $n \geq 2$. This is regarded as an analogue of Euler's formula $\zeta_{2}^{\bullet}(1,2 k)=k \zeta(2 k+1)-\frac{1}{2} \sum_{p=2}^{2 k-1} \zeta(p) \zeta(2 k-p+1)$. See also [3] for related calculations.

\section{ACKNOWLEDGEMENT}

The authors would like to thank Professor Masato Wakayama for valuable comments.

\section{REFERENCES}

1. K. Aomoto, Special values of hyperlogarithms and linear difference schemes, Illinois J. Math. 34 (1990), no. 2, 191-216. MR1046562 (91f:33012)

2. T. Arakawa and M. Kaneko, On multiple L-values, J. Math. Soc. Japan 56 (2004), no. 4, 967-991. MR2091412 (2006b:11108)

3. J. M. Borwein, I. J. Zucker and J. Boersma, The evaluation of character Euler double sums, Ramanujan J. 15 (2008), 377-405. MR.2390277 (2009a:11187)

4. V.G. Drinfel'd, On quasitriangular quasi-Hopf algebras and a group closely connected with $\operatorname{Gal}(\overline{\mathbb{Q}} / \mathbb{Q})$, Leningrad Math. J. 2 (1991), 829-860. MR1080203 (92f:16047)

5. I. G. Macdonald, Symmetric Functions and Hall Polynomials, Second Edition, The Clarendon Press, Oxford Univ. Press, New York, 1995. MR1354144 (96h:05207)

6. T. Ichinose and M. Wakayama, Zeta functions for the spectrum of the non-commutative harmonic oscillators, Commun. Math. Phys. 258 (2005), 697-739. MR2172015 (2006g:81055)

7. T. Ichinose and M. Wakayama, Special values of the spectral zeta function of the noncommutative harmonic oscillator and confluent Heun equations, Kyushu J. Math. 59 (2005), 39-100. MR2134054(2006a:11114)

8. K. Kimoto, Higher Apéry-like numbers arising from special values of the spectral zeta function for the non-commutative harmonic oscillator, preprint, arXiv:0901.0658

9. K. Kimoto and M. Wakayama, Apéry-like numbers arising from special values of spectral zeta functions for non-commutative harmonic oscillators, Kyushu J. Math. 60 (2006), 383-404. MR 2268243 (2007h:11108)

10. K. Kimoto and M. Wakayama, Elliptic curves arising from the spectral zeta function for noncommutative harmonic oscillators and $\Gamma_{0}(4)$-modular forms, The Conference on $L$-Functions, 201-218, World Sci. Publ., Hackensack, NJ, 2007. MR2310296 (2008g:11144)

11. M. Kontsevich and D. Zagier, Periods. Mathematics unlimited-2001 and beyond, 771-808, Springer, Berlin, 2001. MR:1852188(2002i:11002)

12. S. Muneta, On some explicit evaluations of multiple zeta-star values, J. Number Theory $\mathbf{1 2 8}$ (2008), 2538-2548. MR2444209

13. H. Ochiai, A special value of the spectral zeta function of the non-commutative harmonic oscillators, Ramanujan J. 15 (2008), no. 1, 31-36. MR 2372790(2008m:11179)

14. Y. Ohno and D. Zagier, Multiple zeta values of fixed weight, depth, and height, Indag. Math. 12 (2001), 483-487. MR1908876 (2003e:11094) 
15. A. Parmeggiani, Introduction to the spectral theory of non-commutative harmonic oscillators, COE Lecture Note, 8. Kyushu University, The 21st Century COE Program "DMHF", Fukuoka, 2008. MR2381448 (2008m:35254)

16. A. Parmeggiani and M. Wakayama, Oscillator representations and systems of ordinary differential equations, Proc. Natl. Acad. Sci. USA 98 (2001), 26-30. MR1811870 (2002d:81058)

17. A. Parmeggiani and M. Wakayama, Non-commutative harmonic oscillators. I, II; Corrigenda and remarks to I, Forum. Math. 14 (2002), 539-604, 669-690; ibid 15 (2003), 955-963. MR.1900173 (2003g:34186a), MR.1924773 (2003g:34186b)

18. V.S. Varadarajan, Euler and his work on infinite series, Bull. Amer. Math. Soc. (New Series) 44 (2007), no. 4, 515-539. MR.2338363 (2008g:01012)

19. Y. Yamasaki, Evaluations of multiple Dirichlet L-values via symmetric functions, preprint, arXiv:0712.1639

20. D. Zagier, Values of zeta functions and their applications, First European Congress of Mathematics, Vol. II (Paris, 1992), 497-512, Progr. Math. 120, Birkhäuser, Basel, 1994. MR1341859 (96k:11110)

Department of Mathematical Sciences, University of the Ryukyus, Senbaru, NishiHARA, OKINAWA, 903-0231 JAPAN

E-mail address: kimoto@math.u-ryukyu.ac.jp

Faculty of Mathematics, Kyushu University, Hakozaki, Fukuoka, 812-8581 Japan

Current address: Graduate School of Science and Engineering, Ehime University, Bunkyo-cho, Matsuyama, 790-8577 Japan

E-mail address: yamasaki@math.sci.ehime-u.ac.jp 